\title{
Supporting Information for \\ Light-harvesting Properties and Morphology of Porphyrin Nanostructures Depend on Ionic Species Inducing Aggregation
}

\author{
Christopher W. Leishman and Jeanne L. McHale*
}

Department of Chemistry, Box 644630, Washington State University, Pullman, Washington 99164, United States
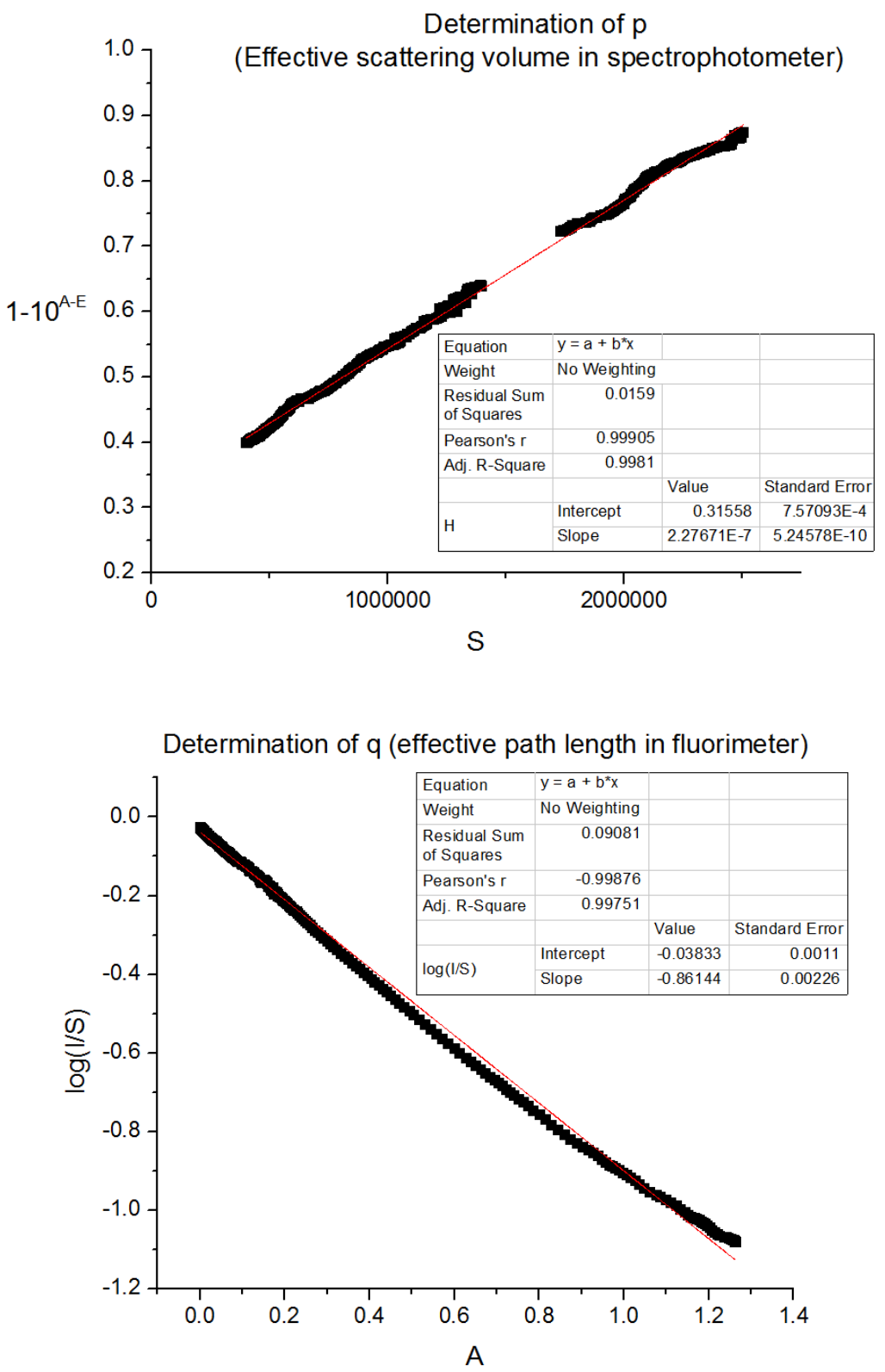

Figure S1. Derivation of extinction and scattering correction parameters. 


\section{MATLAB codes for processing extinction and scattering spectra 1. Generating corrected absorption and RLS spectra from raw extinction and scattering data.}

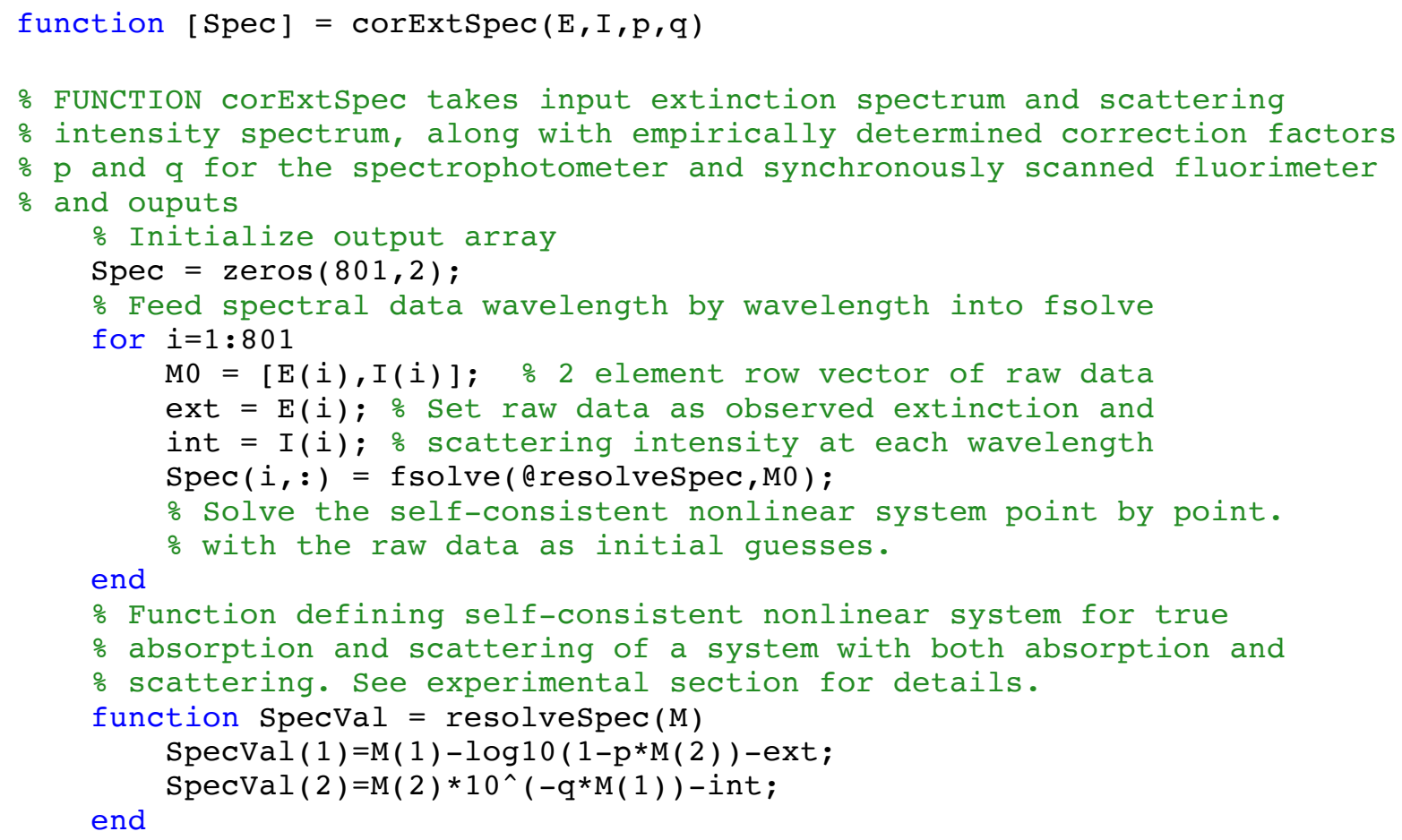

end

\section{Rescaling sample spectra to represent same concentration of aggregated porphyrin.}

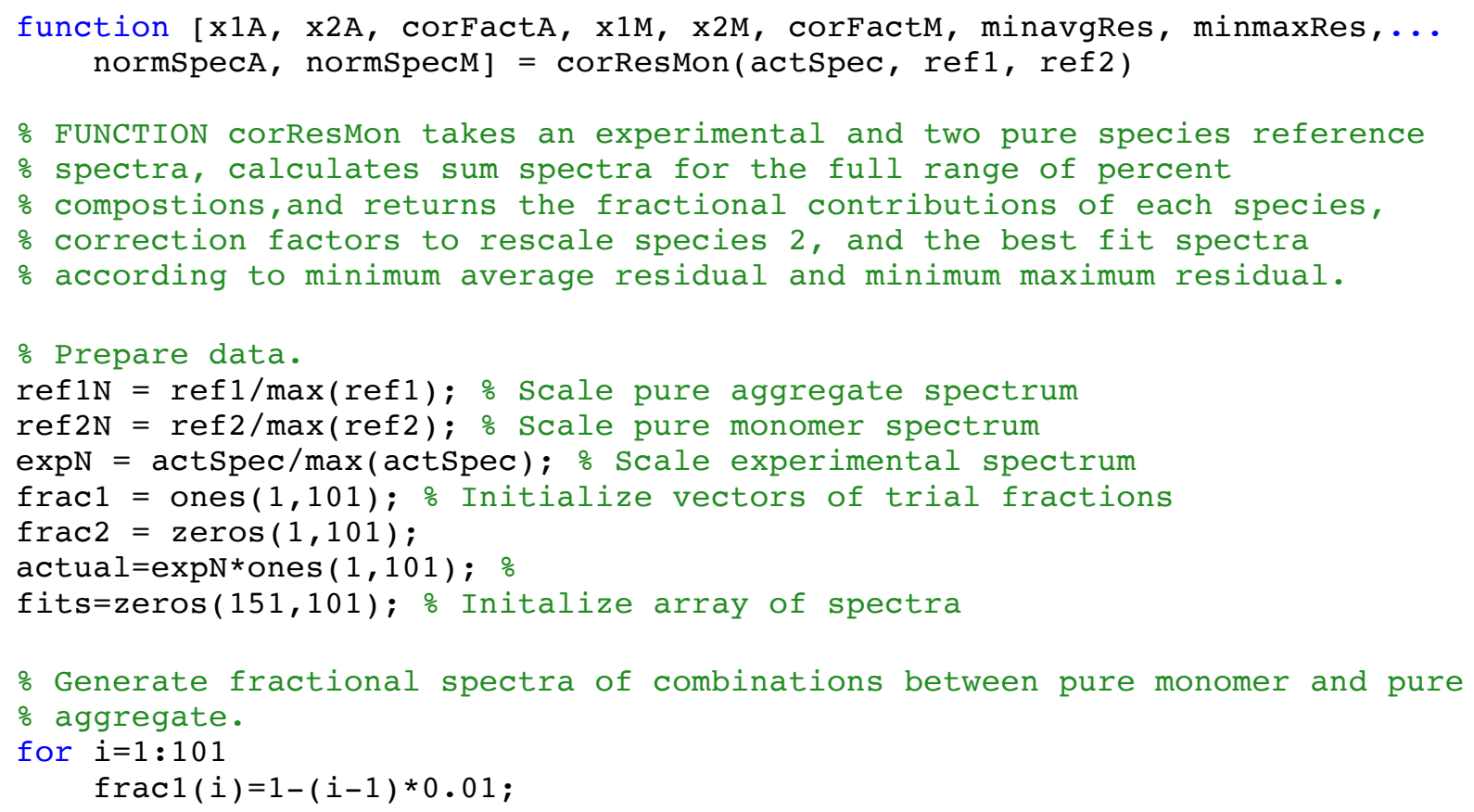




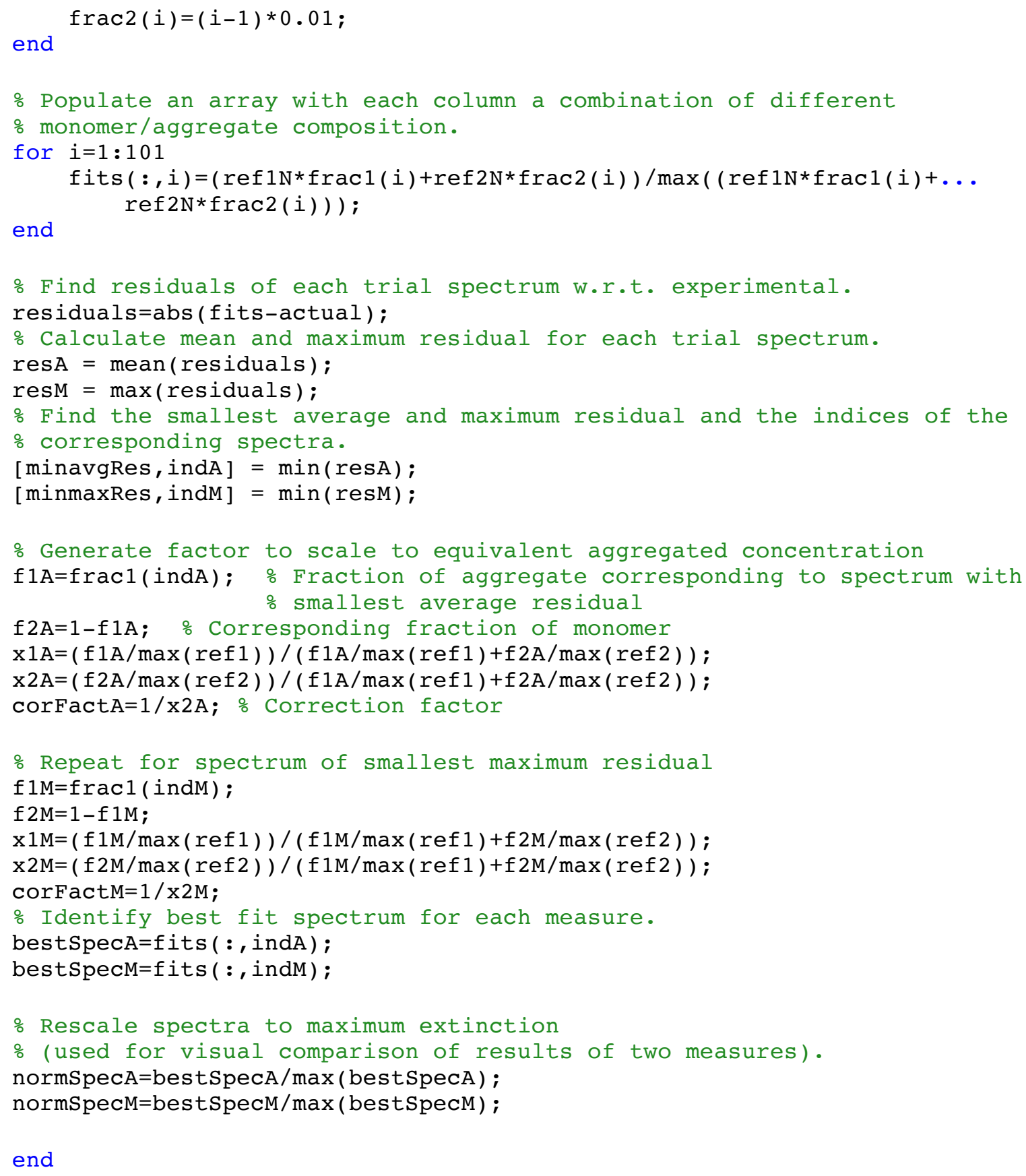



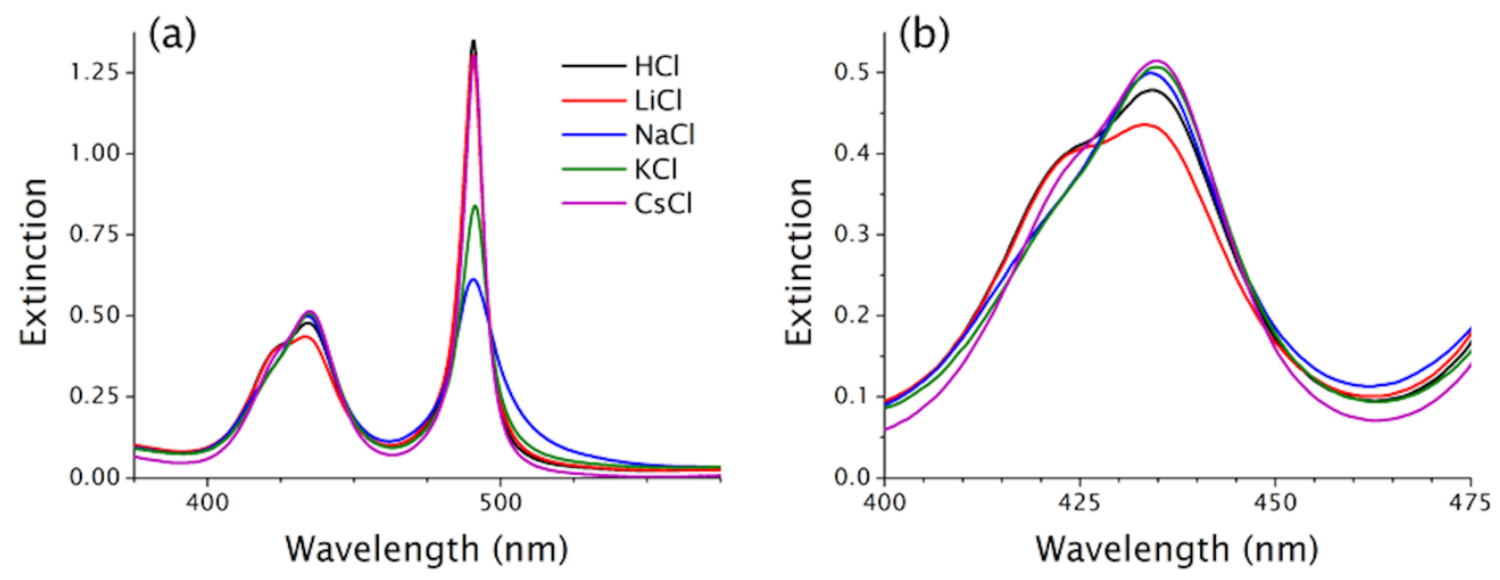

Figure S2. Raw extinction in Soret region (a) and close up of $\mathrm{H}$ band and residual monomer region (b). Solutions are $5 \mu \mathrm{M}$ TSPP in $0.75 \mathrm{M} \mathrm{HCl}$ (black), $\mathrm{LiCl}$ (red), $\mathrm{NaCl}$ (blue), $\mathrm{KCl}$ (green), and $\mathrm{CsCl}$ (purple).
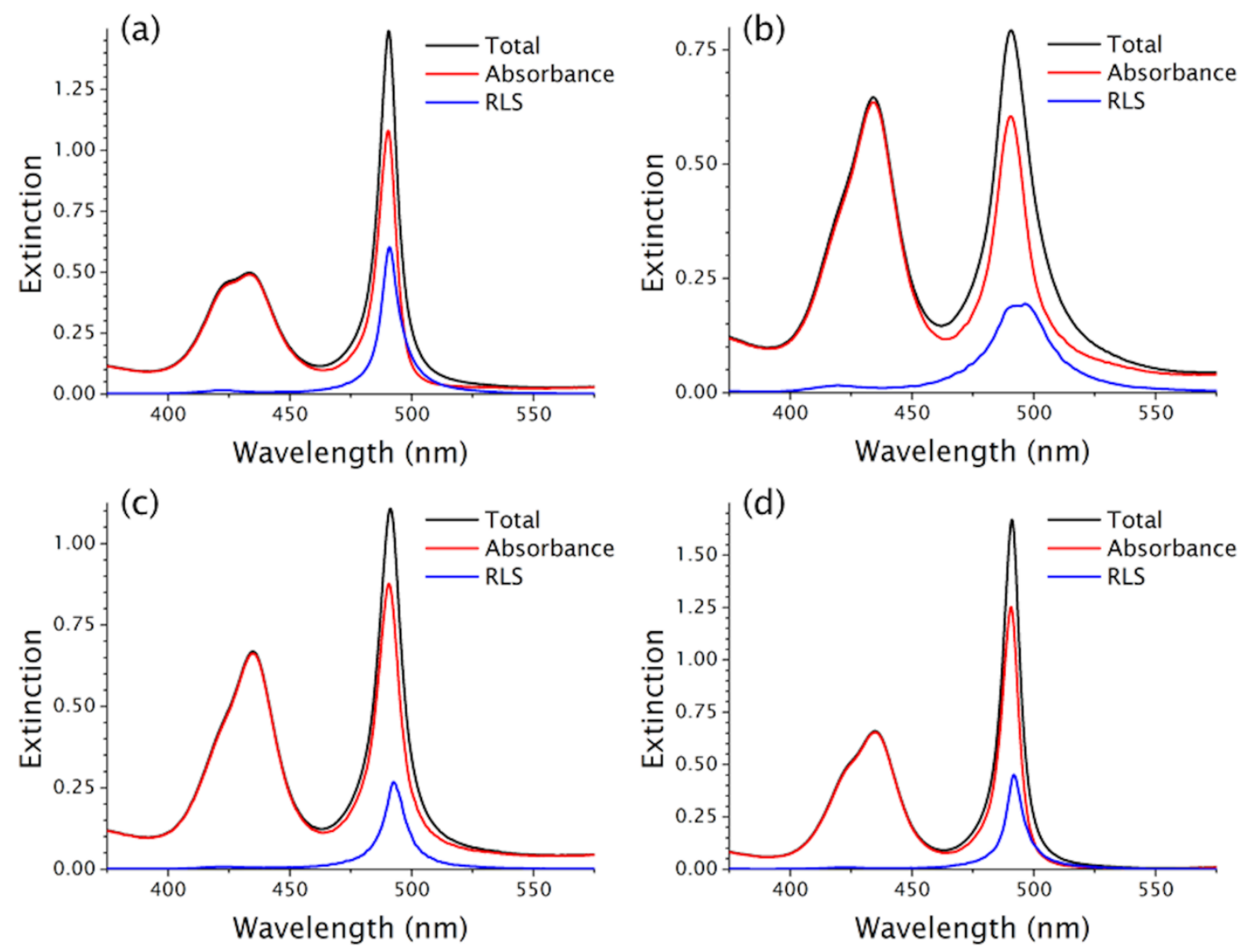

Figure S3. Detail plots of the J band region showing total extinction, absorbance, and RLS (scattering) extinction (black, red, and blue, respectively) for aggregate samples from $5 \mu \mathrm{M} \mathrm{TSPP}$ in $0.75 \mathrm{M} \mathrm{LiCl}$ (a), $\mathrm{NaCl}$ (b), $\mathrm{KCl}$ (c), and $\mathrm{CsCl}(\mathrm{d})$. 


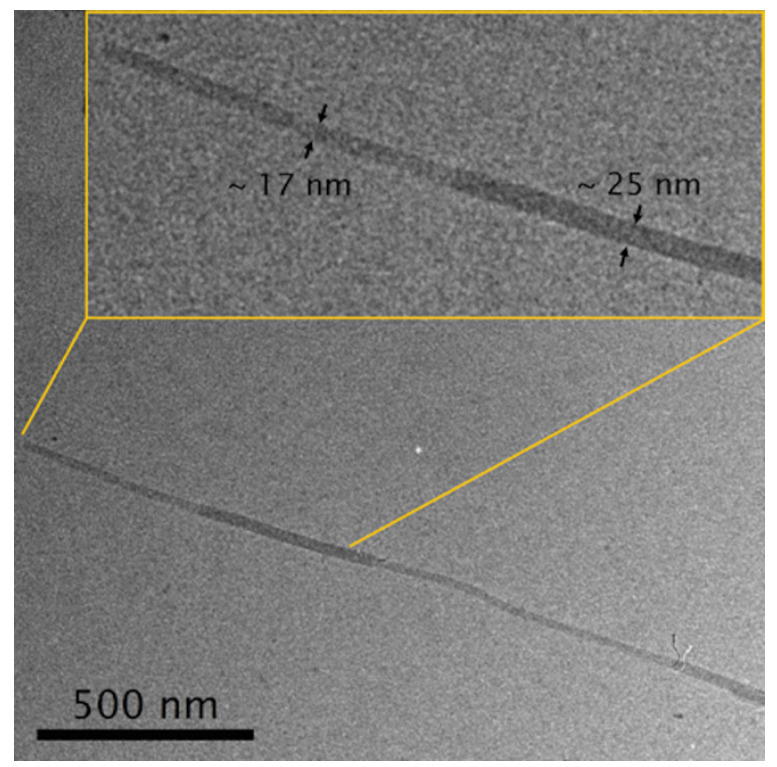

Figure S4. TEM micrograph of a single nanotubular aggregate deposited from $5 \mu \mathrm{M}$ TSPP in $0.75 \mathrm{M} \mathrm{HCl}$. The two locations indicated have widths consistent with intact section $(\sim 17 \mathrm{~nm})$ and a collapsed section $(\sim 25 \mathrm{~nm})$.

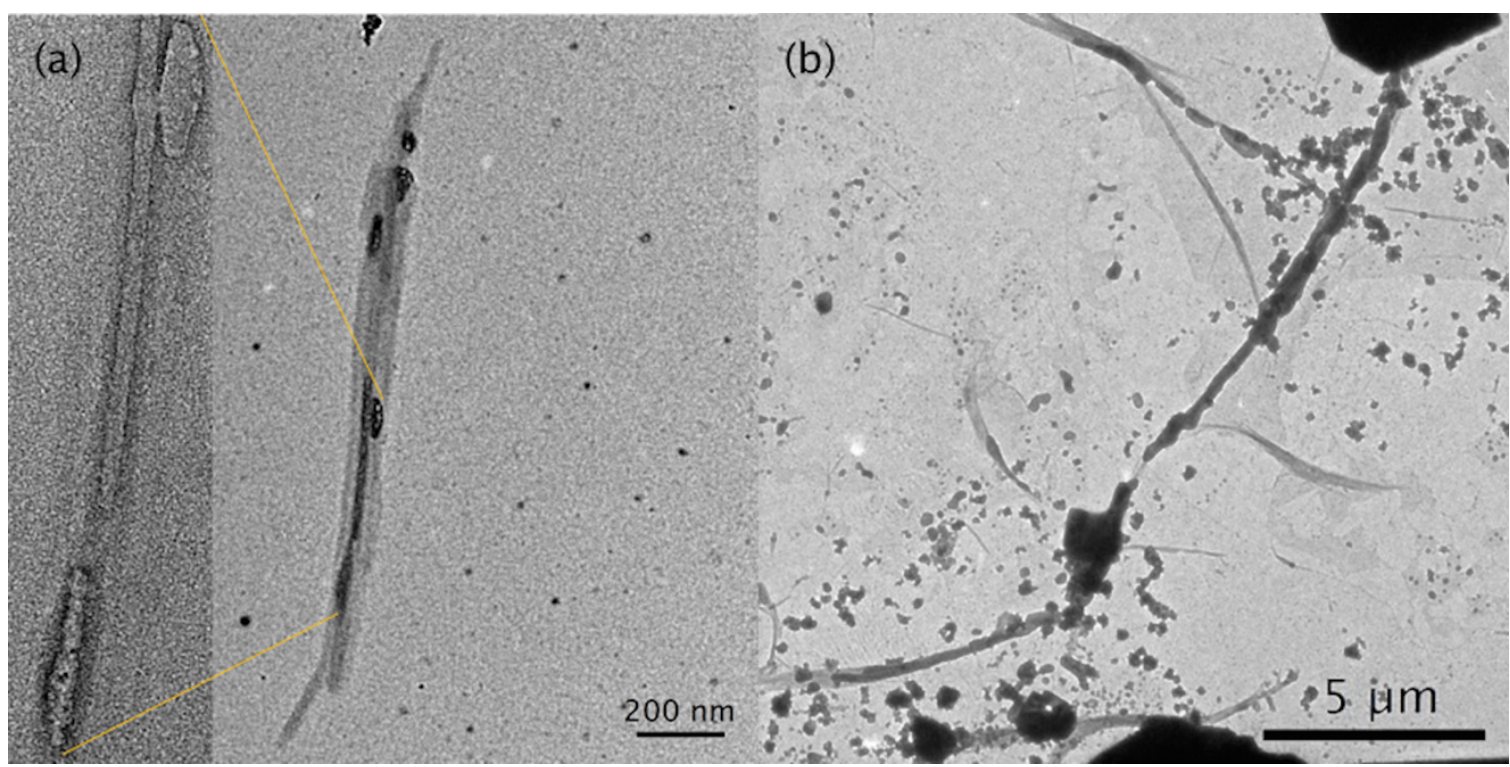

Figure S5. TEM micrographs of aggregates deposited from $0.75 \mathrm{M} \mathrm{KCl}$, (a) showing a small bundle of several nanotubes and (b) showing large amount of microcrystalline $\mathrm{KCl}$. 

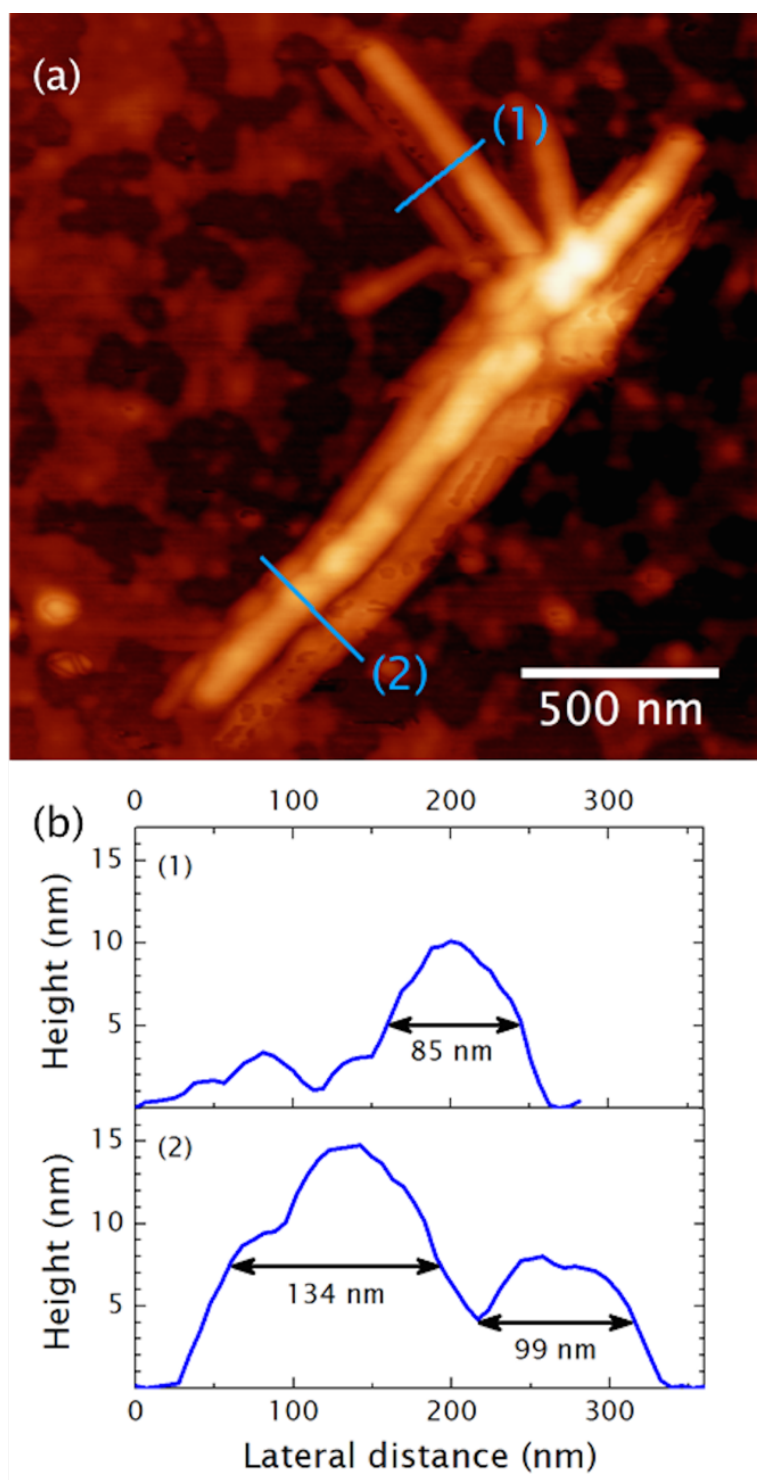

Figure S6. (a) AFM images of aggregates deposited from $5 \mu \mathrm{M}$ TSPP in $0.75 \mathrm{M} \mathrm{KCl}$. (b) Cross-sectional height profiles at the locations labeled in (a). 


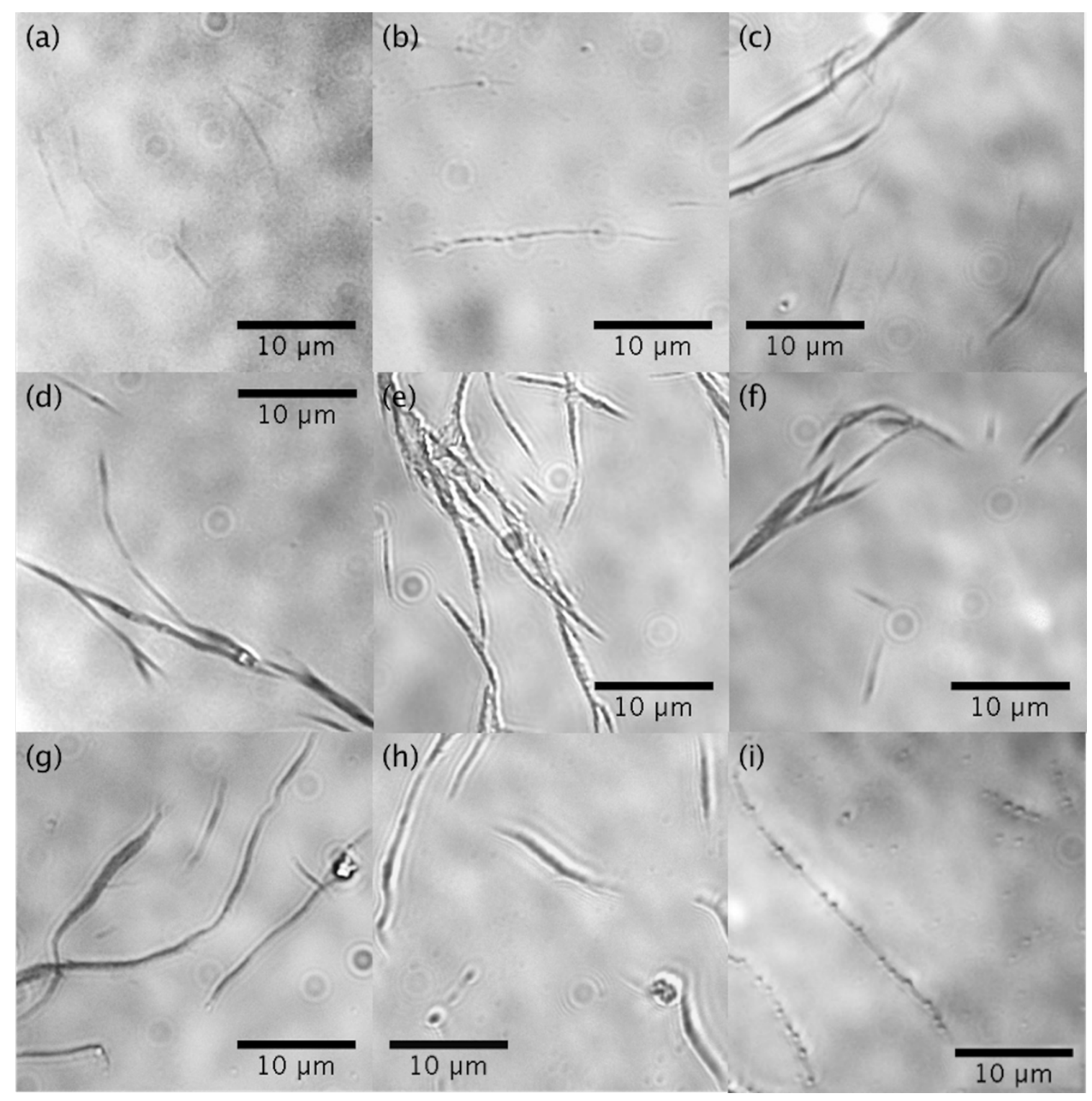

Figure S7. Optical images of aggregates from different samples and drying methods on glass coverslips. All samples have the same concentrations of TSPP $(5 \mu \mathrm{M})$ and either acid or salt $(0.75 \mathrm{M})$ as used for the spectroscopy, AFM, and TEM experiments. Methods of drying are given as A: Air drying at ambient temperature; $\mathrm{H}$ : Air dried in oven heated to $\sim 150{ }^{\circ} \mathrm{C}$; S: Spin dried after allowing drop to stand for 5 minutes. (a) HCl-A (b) LiCl-H (c) LiCl-S (d) NaCl-A (e) NaCl-H (f) NaCl-S (g) KCl-A (h) KCl-S and (i) $\mathrm{CsCl}-\mathrm{S}$. Note that images are slightly defocused to provide contrast for features with diffraction-limited widths, especially in (a) and (i). 

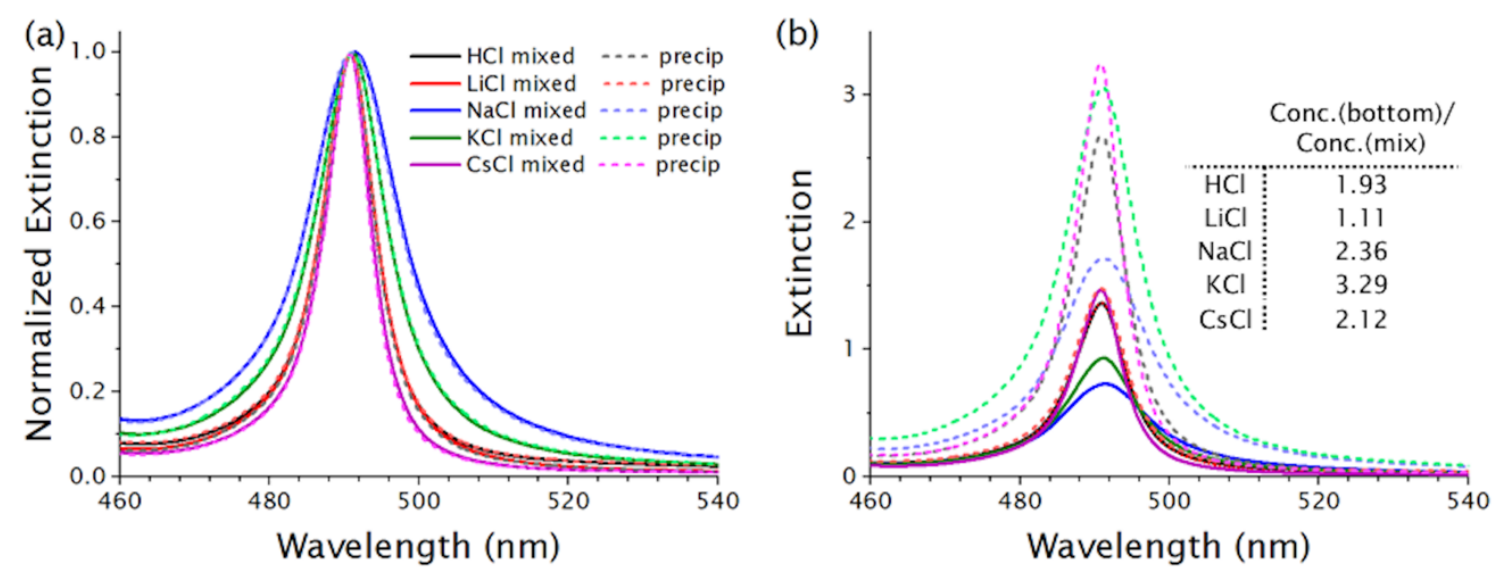

Figure S8. (a) Normalized $\mathrm{J}$ band extinction spectra ( $5 \mu \mathrm{M}$ TSPP/0.75 $\mathrm{M} \mathrm{HCl}$ or salt). Solid lines: thoroughly mixed solutions; Dashed lines: bottom $1.5 \mathrm{~mL}$ of undisturbed sample after standing for 3 days, containing flocculated/precipitated aggregates. (b) Non-normalized J band extinction spectra of sample series, with inset figure giving concentration ratio of solution containing flocculate from bottom of vials to the mixed solution, based on integration of $J$ band integrated from the minimum at $18000 \mathrm{~cm}^{-1}(556 \mathrm{~nm})$ to the minimum at $21500 \mathrm{~cm}^{-1}(465 \mathrm{~nm})$. The mixed solution spectra were taken immediately after returning the aliquot of flocculate to the sample vial and manually swirling to mix.

\section{Theoretical considerations for interpretation of RLS spectra}

Theoretical discussion of RLS and its relationship to excitonic coherence in the literature is limited. ${ }^{1,2}$ These models implicitly assume a single excitonic transition. We can analyze the more general case of multiple, possibly interfering excitons through a Kramers-Heisenberg-Dirac expression for the transition polarizability for Rayleigh scattering:

$$
\left(\alpha_{\rho \sigma}\right)_{i i}=\hbar^{-1} \sum_{n} \frac{\mu_{\rho, i n} \mu_{\sigma, n i}}{\omega_{i n}-\omega_{L}+i \Gamma_{n}}
$$

Here we use a short hand notation for the dipole moment matrix elements, for example the element $\mu_{\rho, \text { in }}=\left\langle i\left|\mu_{\rho}\right| n\right\rangle$ connecting the initial state $i$ to the intermediate state $n$ via dipole interaction with a field of polarization $\rho$ and frequency $\omega_{L}$. The transition frequency $\omega_{\text {in }}$ and the incident light. The quantity $\Gamma_{n}$ is a generalized phenomenological decay rate for the intermediate state $n$. Each $i$ and each $n$ should be understood as a complete set of quantum numbers specifying the vibronic state, including the electronic state and the vibrational levels of all normal modes. We have left out the anti-resonance terms containing $\omega_{i n}+\omega_{L}$ because these are negligible near resonance. 
The Rayleigh scattering cross section is proportional to the sum of square moduli over all $i \rightarrow i$ transitions in the thermal population given by the distribution function $P_{e q, i}$.

$$
\sigma_{R L S} \propto\left|\left(\alpha_{\rho \sigma}\right)_{i i}\right|^{2}=\hbar^{-2} \sum_{i} P_{e q, i} \sum_{n, m}\left(\frac{\left|\mu_{i n}\right|^{2}}{\omega_{i n}-\omega_{L}+i \Gamma_{n}}\right)\left(\frac{\left|\mu_{i m}\right|^{2}}{\omega_{i m}-\omega_{L}-i \Gamma_{m}}\right)
$$

We have ignored the polarization dependence of transition moments for simplicity, as we are not presently concerned with depolarization in RLS. For $N_{c}$ coupled monomer units, there are in principle $N_{c}$ excitonic transitions. The phase relation between contiguous monomers is typically described by the excitonic wavenumber $k$, which runs from 0 to $\pi$ in increments of $\pi / N_{c}$. For each linear dimension of coherently coupled chromophores, this quantity can be expressed as ${ }^{3}$

$$
\mu_{k}=\left(\frac{2}{N_{c}+1}\right)^{1 / 2} \sum_{n}^{N_{c}} \mu_{m o n} \sin \left(\frac{\pi k n}{N_{c}+1}\right)
$$

It has been shown that the $k=0$ exciton with all monomers exactly in phase carries all the oscillator strength in the absence of disorder, ${ }^{3}$ as intuitively expected for transition from a symmetric ground state. The corresponding transition moment approaches square root dependence on the coherence number for $N_{c} \gtrsim 20$. $^{1}$

Referring to equation (S2), we can further analyze the lineshapes of the RLS spectra. For two overlapping excitonic transitions originating in the same ground state, with transition moments $\mu_{i A}$ and $\mu_{i B}$ scale, at maximum, as $N_{C, A}^{1 / 2}$ and $N_{C, B}^{1 / 2}$ respectively. We assume that the exciton decay rates $\Gamma_{A, B}$ are approximately equal. Then the RLS scattering cross section (or the intensity) scales at most as

$$
\begin{aligned}
\sigma_{R L S} \propto \sum_{n, m=A, B} & \left(\frac{N_{C, n}}{\Delta_{i n}+i \Gamma_{n}}\right)\left(\frac{N_{C, m}}{\Delta_{i m}-i \Gamma_{m}}\right) \\
& =\left(\frac{N_{C, A}^{2}}{\Delta_{i A}^{2}+\Gamma_{A}^{2}}\right)+\left(\frac{N_{C, A} N_{C, B}}{\Delta_{i A} \Delta_{i B}+i \Gamma_{A} \Delta_{i B}-i \Gamma_{B} \Delta_{i A}+\Gamma_{A} \Gamma_{B}}\right) \\
& +\left(\frac{N_{C, A} N_{C, B}}{\Delta_{i A} \Delta_{i B}+i \Gamma_{B} \Delta_{i A}-i \Gamma_{A} \Delta_{i B}+\Gamma_{A} \Gamma_{B}}\right)+\left(\frac{N_{C, B}^{2}}{\Delta_{i B}^{2}+\Gamma_{B}^{2}}\right)
\end{aligned}
$$


Here we have introduced the detunings $\Delta_{i n}=\omega_{\text {in }}-\omega_{L}$, etc. In the case of two transitions of the same energy, the maximum possible intensity (integrated over $\omega_{L}$ ) would occur, with the resonance denominators all equal for all $\omega_{L}$ and the intensity proportional to $\left(N_{C, n}+N_{C, m}\right)^{2}$. Otherwise, contributions from the frequency region between the two transition center frequencies have (-) sign (because the detunings there have opposite signs) and partially cancel contributions with $(+)$ sign from outside this frequency region, resulting in an integrated intensity that scales less rapidly than $\left(N_{C, n}+N_{C, m}\right)^{2}$. Additionally, the effective transition moments scale less rapidly than $N_{C, n}^{1 / 2}$ for any excited state $n$ with $k>0$, and the interferences less rapidly than $N_{C, A} N_{C, B}$ for transitions of differing optical polarization. We thus conclude that $\left(N_{C, n}+N_{C, m}\right)^{2}$ is an upper bound on the dependence of integrated RLS intensity on spatial coherence for two overlapping excitonic transitions. This readily generalizes to larger numbers of overlapping excitons. As the RLS intensity scales no more rapidly with coherence than this, we can say that the sum of coherence numbers of all excitonic transitions, averaged over individual aggregates, must be larger when a greater integrated RLS intensity is observed.

\section{References}

(1) Parkash, J.; Robblee, J. H.; Agnew, J.; Gibbs, E.; Collings, P.; Pasternack, R. F.; de Paula, J. C. Depolarized Resonance Light Scattering by Porphyrin and Chlorophyll a Aggregates. Biophys. J. 1998, 74, 2089-2099.

(2) Collings, P. J.; Gibbs, E. J.; Starr, T. E.; Vafek, O.; Yee, C.; Pomerance, L. A.; Pasternack, R. F. Resonance Light Scattering and Its Application in Determining the Size, Shape, and Aggregation Number for Supramolecular Assemblies of Chromophores. J. Phys. Chem. B 1999, 103, 8474-8481.

(3) Alden, R. G.; Lin, S. H.; Blankenship, R. E. Theory of Spectroscopy and Energy Transfer of Oligomeric Pigments in Chlorosome Antennas of Green Photosynthetic Bacteria. J. Lumin. 1992, $51,51-66$. 